\title{
LA IMPORTANCIA DE LA EMOCIÓN EN EL APRENDIZAJE
}

\author{
Aránzazu Elizondo Moreno \\ areli7815@hotmail.com \\ UNIVERSIDAD POLITÉCNICA DE CARTAGENA \\ José Víctor Rodríguez Rodríguez \\ Jvictor.rodriguez@upct.es \\ Dpto. de Tecnologías de la Información y las Comunicaciones \\ UNIVERSIDAD POLITÉCNICA DE CARTAGENA \\ Ignacio Rodríguez Rodríguez \\ ignacio.rodriguez1@um.es \\ Dpto. de Ingeniería de la Información y las Comunicaciones \\ UNIVERSIDAD DE MURCIA
}

Recibido: 23 de octubre de 2017 Aceptado: 20 de noviembre de 2018

DOI: http://dx.doi.org/10.15366/didacticas2018.19.003

\section{Resumen}

En este trabajo, se aborda la importancia de las emociones en la adquisición de conocimiento a partir de las últimas investigaciones llevadas a cabo al respecto en el campo de la neurociencia y la psicología. En este sentido, se analiza la influencia de los estados emocionales negativos y positivos en el aprendizaje, así como la importancia de la autorregulación emocional y la motivación en la adquisición de conocimiento. De esta manera, se propone la consideración de todas estas cuestiones en el proceso de elaboración de nuevas y más eficientes metodologías de enseñanza-aprendizaje en pos de una evolución y mejora de la práctica educativa.

Palabras Clave: Pedagogía; aprendizaje; metodología; emoción; motivación.

\begin{abstract}
In this work, the importance of emotions in the acquisition of knowledge, based on the latest research carried out in neuroscience and psychology on that subject, is analyzed. In this sense, the influence of negative and positive emotional states in learning as well as the importance of emotional autoregulation and motivation in the acquisition of knowledge are studied. This way, the consideration of all these issues in the elaboration process of new and more efficient teaching-learning methodologies is proposed in order to improve the educational practice.
\end{abstract}

Keywords: Pedagogy; learning; methodology; emotion; motivation. 


\section{1.- INTRODUCCIÓN}

Cuando, hace más de 2000 años, Platón enunció la cita "todo aprendizaje tiene una base emocional" quizá no fuera consciente de, hasta qué punto, estaba adelantándose a las conclusiones que se derivan de las evidencias que arrojan recientes estudios llevados a cabo en los campos de la neurobiología y la educación. Ciertamente, más allá de las teorías anteriores que consideraban la emoción y la cognición como actividades completamente independientes, el aprendizaje comienza a ser visto como una interacción sinérgica de elementos cognitivos, emocionales y fisiológicos en la que las emociones -capaces de re-esculpir literalmente nuestro tejido neuronal- se muestran como una parte muy importante y poderosa del proceso de adquisición de conocimiento. Por tanto, en este artículo, se aborda la importancia de las emociones en dicho proceso teniendo en cuenta las últimas investigaciones llevadas a cabo al respecto en el campo de la neurociencia y la psicología. De esta manera, se analiza la influencia de los estados emocionales negativos y positivos en el aprendizaje, así como la importancia tanto de la llamada autorregulación emocional como de la motivación en su desarrollo.

\section{2.- LOS ESTADOS EMOCIONALES NEGATIVOS EN EL APRENDIZAJE}

Está comprobado que los estados emocionales negativos, surgidos a partir de situaciones de miedo o estrés, propician una activación de la amígdala que deriva en una liberación de adrenalina, noradrenalina y glucocorticoides (cortisol). Así mismo, la presencia de estas hormonas provoca, a su vez, una serie de eventos corporales como el aumento de la tasa de pulsaciones y de transpiración (Damasio, 1994; Le Doux, 2000). De esta manera, si bien es cierto que un nivel leve o moderado de estrés (que supone valores bajos y medios de cortisol) es esencial para una adaptación óptima a los desafíos ambientales y puede favorecer el rendimiento cognitivo en la medida que provoca la elevación del nivel general de alerta (Borod, 2000; Whalen, 1998), situaciones de estrés intenso (en las que se liberan niveles altos de cortisol) tienen un efecto nocivo sobre el aprendizaje y la memoria, bloqueando el proceso cognitivo, y pudiendo suponer, si el estrés se prolonga en el tiempo, incluso el deterioro de las neuronas del hipocampo, estructura que resulta esencial para adquirir conocimiento (McEwen y Sapolsky, 1995). Pueden imaginarse, por tanto, las nefastas consecuencias a nivel de aprendizaje que tendrá -en el ámbito académico- sobre un estudiante una eventual atmósfera de miedo o estrés en el aula propiciada por profesores agresivos, acoso escolar (bullying) o materiales educativos incomprensibles. Además, ciertas ideas, en cierto modo imperantes en nuestra sociedad, que tienen que ver con fomentar la competitividad extrema, así como con la necesidad ineludible de alcanzar el éxito suponen, sin duda, una fuente añadida de elementos estresores en el aprendiz. 


\section{3.- LOS ESTADOS EMOCIONALES POSTIVOS EN EL APRENDIZAJE}

Por el contrario, los estados emocionales positivos activan los llamados núcleos dopaminérgicos liberando dopamina que, a su vez, estimula, en los ganglios basales, la producción de neuropéptidos (también llamados opiáceos endógenos por generar un efecto similar al que provoca el opio). De esta forma, la presencia de los anteriores neuromoduladores fortalece las sinapsis que estén activas en ese momento, favoreciendo el aprendizaje (Wise, Spinder, De Wit y Gerber 1978; Wise, 1982). Es decir, parece claro que, en situaciones de bienestar emocional, aumenta la eficiencia del proceso cognitivo.

\section{4.- APRENDIZAJE EMOCIONAL}

Un claro ejemplo del papel que juegan las emociones en la adquisición de conocimiento lo constituye el denominado aprendizaje emocional, en el que, a través de la asociación de un determinado estímulo con una emoción (condicionamiento), surge ese aprendizaje $y$, por tanto, dichos estímulos dejan de ser emocionalmente neutros para adquirir un valor o significado emocional (Smith y Kosslyn, 2007). Existen diversos tipos de aprendizaje emocional, como el condicionamiento clásico, demostrado por primera vez por Iván Pávlov, donde un estímulo neutro se presenta repetidas veces junto a un estímulo con carga emocional hasta que llega un momento en el que el primero produce, por sí sólo -en ausencia del segundo-, la experiencia emocional de este último. Además, también puede obtenerse aprendizaje emocional a través del denominado condicionamiento instrumental, u operante, introducido por Edward Thorndike, que consiste en el desarrollo de nuevas conductas en función de sus consecuencias emocionales. Es decir, si la emoción obtenida es placentera, la conectividad neural subyacente al suceso experimentado se fortalecerá (se aprenderá) y el sujeto tratará de repetirla con mayor frecuencia. Por el contrario, si la emoción experimentada es displacentera, la conectividad subyacente también se fortalecerá, pero se intentará evitar el suceso. Otros ejemplos de aprendizaje emocional que, sin embargo, no requieren, en este caso, la presencia de una experiencia emocional son la instrucción (se adjudica significado emocional a estímulos inicialmente neutros a partir de información recibida de otras personas), la observación (el aprendizaje surge al observar la respuesta emocional de otras personas ante los estímulos) y la exposición (se desarrolla una preferencia hacia los objetos, lugares o situaciones familiares).

\section{5.- AUTORREGULACIÓN EMOCIONAL Y APRENDIZAJE}

Por otra parte, el hecho de saber influir sobre las propias emociones, o la llamada autorregulación emocional (Thompson, 1994; Gros, 2003), es conocido que representa un componente esencial de la competencia necesaria para una interacción exitosa con otros en situaciones de estrés, pero lo interesante, es que también se ha mostrado como 
una habilidad clave para favorecer un aprendizaje eficaz. En primer lugar, porque las emociones pueden actuar sobre los procesos psicológicos responsables de focalizar la atención o de resolver problemas (Cole, Martin y Dennis, 2004) pero, además, porque la capacidad de autocontrol de los propios impulsos en niños - con el fin de postergar una gratificación- ha resultado ser un poderoso indicativo de un exitoso rendimiento académico ulterior (Carlton y Winsler, 1999). Esta última afirmación ha sido respaldada por diferentes estudios como el basado en un experimento en el que se encomendó a una serie de niños la tarea de resistirse a comer una porción de tarta con la promesa de obtener dos si habían logrado sobreponerse a la tentación cuando regresara el investigador. El tiempo de demora durante el cual el niño tuvo éxito en resistir el impulso de comerse la primera porción de tarta estuvo significativamente correlacionado con un exitoso rendimiento académico posterior, medido como la habilidad de afrontar la frustración, el estrés, la perseverancia en la tarea y la concentración (OECD, 2002).

Esta autorregulación emocional, entendida en su vertiente de aptitud para motivarse a uno mismo, sería una de las dimensiones que abarcaría la denominada inteligencia emocional (Salovey y Mayer, 1990), que se define como la capacidad de identificar, entender y manejar las emociones correctamente en pos de facilitar las relaciones con los demás (empatía), la consecución de metas y objetivos, el manejo del estrés o la superación de obstáculos. Se ha demostrado que el desarrollo de este tipo de inteligencia o competencia aumenta la capacidad de aprendizaje del cerebro e incide muy positivamente en el rendimiento académico (Goleman, 1996).

\section{6.- MOTIVACIÓN Y APRENDIZAJE}

Otro elemento que se encuentra estrechamente vinculado con las emociones y que resulta crucial para favorecer el aprendizaje es la motivación, que puede ser descrita como la "fuerza o acción resultante de los componentes emocionales" (Borod, 2000). La motivación está íntimamente relacionada con las emociones porque refleja hasta qué punto un organismo está preparado para actuar física y mentalmente de una manera focalizada, y la respuesta emocional constituye la forma en que el cerebro evalúa si actuar, o no, sobre las cosas -aproximarse a ellas, si son placenteras, o evitarlas, si son desagradables-. Por tanto, se puede afirmar que los sistemas emocionales crean motivación, y ésta, en la medida que predispone a la acción inducida y mantenida por esas emociones, propicia el aprendizaje.

Es posible diferenciar entre dos tipos principales de motivación: la motivación extrínseca (vinculada a factores externos) y la motivación intrínseca (vinculada a factores internos). Mientras que la motivación extrínseca se consigue por medio de referentes para la acción exteriores al individuo - por ejemplo, castigos y recompensas (McGraw, 1978) - la motivación intrínseca responde a la sencilla pretensión de satisfacer las necesidades y los deseos internos. En este sentido, cabe resaltar que el sistema educativo tradicional se ha basado fundamentalmente en la motivación extrínseca, desdeñando el 
potencial para el aprendizaje de una motivación intrínseca que, aprovechando la sensación sumamente placentera que proporciona la obtención de conocimiento, podría ser el verdadero motor del aprendiz. Ciertamente, a través de ese estado descrito como flujo, en el que el sujeto se encuentra realmente comprometido en búsquedas que le brindan placeres fundamentales sin ninguna promesa de recompensa externa (Csikszentmihalyi, 1990), la motivación intrínseca basada en la indescriptible emoción que se da al comprender algo (una suerte de iluminación) se manifiesta como un poderoso recurso a favor de la búsqueda del aprendizaje. Esta especie de iluminación mencionada correspondiente al momento en el que el cerebro súbitamente realiza una serie de conexiones y reconoce patrones entre la información disponible (comprende) fue acertadamente descrita como el momento "eureka" por Peter Gärdefors, de la Universidad de Lund (Suecia) durante una conferencia en Copenhague, organizada por el CERI (Center for Educational Research and Innovation) y el Laboratorio de Aprendizaje de Dinamarca, en noviembre de 2004. Así, es considerada como una de las sensaciones más placenteras que existen. Sería deseable, pues, que, desde una edad temprana, se fomentara, en el ámbito educativo, el hecho de que el alumnado experimente la gratificación que acompaña a la comprensión de información -que, a su vez, da lugar a conocimiento- dado que, una vez vivida esa experiencia emocional, es muy probable que intente repetirse. El desafío es encontrar cómo darle un propósito al aprendizaje y cómo estimular el impulso interno del deseo de aprender.

\section{7.- CONCLUSIONES}

A partir de todas las evidencias científicas anteriormente expuestas en relación a la importancia de las emociones en el aprendizaje, no sólo la sabiduría encerrada en la milenaria cita platónica que abría este trabajo se revela como increíblemente acertada, sino que la consideración de los hallazgos que emergen de la neurociencia debería ser algo ineludible en la elaboración de nuevas y más eficientes metodologías de enseñanzaaprendizaje - a través de lo que se ha venido a llamar MBE (Mind, Brain and Education)en pos de una evolución y mejora de la práctica educativa.

\section{BIBLIOGRAFÍA}

BOROD, J. C. (2000). The neuropsychology of emotion, Nueva York: Oxford University Press.

CARLTON, M.P. y A. WINSLER (1999). School Readiness: The Need for a Paradigm Shift, School Psychology Review, vol. 28, núm. 3, pp. 338-352. 
COLE, P.M., S.E martin y T.A. Dennis (2004). Emotion Regulation as a Scientific Construct: Methodological Challenges and Directions for Child Development Research, Child evelopment, vol. 75, núm. 2, pp. 317-333.

CSIKSZENTMIHALYI, M. (1990). Flow: The Psychology of Optimal Experience, Harper and Row, Nueva York.

DAMASIO, A.R. (1994). Descartes' Error: Emotion, Reason, and the Human Brain, G.P. Putnam, Nueva York.

GOLEMAN, D (1996). Inteligencia emocional. Barcelona: Kairós.

GROS, B. (2003). The Impact of Digital Games in Education, First Monday, diario revisado por pares.

LEDOUX, J.E. (2000). Emotion Circuits in the Brain, Annual Review of Neuroscience, vol. 23, pp. 155-184.

MCGRAW, K.O. (1978). The Detrimental Effects of Reward on Performance: A Literature Review and a Prediction Model, en M.R. Lepper y D. Greene (eds.), The Hidden Costs of Reward: New Perspectives on the Psychology of Human Motivation, Lawrence Erlbaum, Hillsdale, NJ, pp. 33-60.

MCEWEN, B.S. y R.M. Sapolsky (1995). Stress and Cognitive Function, Curr Opin Neurobiol, vol. 5, pp. 205-216.

OECD (2002). Primer foro de alto nivel sobre "Mecanismos del cerebro y el aprendizaje temprano", Nueva York, www.oecd.org/dataoecd/40/18/15300896.pdf.

SALOVEY, P. y MAYER, J. D. (1990). Emotional intelligence. Imagination, Cognition and Personality, 9, 185-211.

SMITH, E. E., y KOSSLYN, S. M. Cognitive psychology: Mind and Brain. Nueva York. Prentice Hall.

THOMPSON, R.A. (1994). Emotional Regulation: A Theme In Search Of a Definition, en N.A. Fox (ed.), The Development of Emotion Regulation: Biological and Behavioural Considerations. Monographs of the Society for Research in Child Development, serie núm. 240, vol. 59, núms. 2-3, University of Chicago Press, Chicago, pp. 25-52.

WHALEN, P. J. (1998). Fear, vigilance, and ambiguity: Initial neuroimaging studies of the human amygdala. Current Directions in Psychological Sciences, 7, 177-188.

WISE, R. A. (1982). Neuroleptics and operant behaviour: the anhedonia hypothesis. Behavioral and Brain Sciences, 5, 39-87.

WISE R. A., SPINDER, J., DE WIT, H., GERBER, G. J. (1978). Neuroleptic-induced Aanhedonia@ in rats: pimozide blocks reward quality of food. Science, 201, 262-264. 